\section{Intensive Care Admissions for Severe Chikungunya Virus Infection, French Polynesia}

\section{Adrien Koeltz, Stephane Lastere, Sylvain Jean-Baptiste}

Author affiliations: Taaone Hospital, Papeete, French

Polynesia (A. Koeltz, S. Lastere); Bichat Hospital, Paris, France

(S. Jean-Baptiste)

DOI: https://doi.org/10.3201/eid2404.161536

During the 2014-2015 chikungunya outbreak in French Polynesia, 64 patients with confirmed chikungunya virus infection were admitted into intensive care. Sixty-three were nonpregnant adults; 11 had an atypical form, 21 had severe sepsis or septic shock, and 18 died. These findings indicate that critical illness frequently complicates the course of chikungunya virus infection.

$\mathrm{T}$ he first case of chikungunya virus (CHIKV) infection in French Polynesia (Tahiti) was diagnosed in May 2014; it was imported from Guadeloupe Island in the Caribbean (1). During the outbreak that developed during October 2014-March 2015, $\approx 25 \%$ of the local population $(272,000$ residents) became infected with CHIKV (2). French Polynesia has 2 potential mosquito vectors for CHIKV: Aedes aegypti and A. polynesiensis. Phylogenic analysis showed that the French Polynesia strain of CHIKV belongs to the Asian lineage and is closely related to the strain collected in Guadeloupe and the British Virgin Islands in 2014, showing $99.9 \%$ homology with that strain (3). To describe patient characteristics and clinical courses of chikungunya patients in French Polynesia during 2014-2015, we retrospectively reviewed the medical files of all patients with documented CHIKV infection.

CHIKV infection was defined by the association of compatible symptoms of fever and arthralgia and positive IgM serology or positive blood reverse transcription PCR (RT-PCR). We defined types of CHIKV infection as follows: 1) common form (i.e., only fever or arthralgia); 2) atypical form (i.e., involvement of $\geq 1$ organ systems); and 3) severe form (i.e., failure of $\geq 1$ organ systems or admission to an intensive care unit [ICU]).

We used standard definitions for organ system failures and severe sepsis shock (4). Organ failures were defined by a Sequential Organ Failure Assessment score $\geq 3$ for each organ. Encephalitis was defined in accordance with Position Consensus Statement of the International Encephalitis Consortium criteria (5) and myocarditis in accordance with Consensus Statement of the European Society of Cardiology criteria (6).
During the outbreak, CHIKV was confirmed in 63 adults and one 11-year-old girl (Table). Forty-two patients had positive results for blood RT-PCR, and 22 had positive results for IgM serology. Virus load in serum was high; median load was $7.52 \log _{10}$ copies/mL (interquartile range 3.47-9.39 $\log _{10}$ copies $/ \mathrm{mL}$ ). Of 5 patients with encephalitis symptoms, 3 had positive results for cerebrospinal fluid RT-PCR.

Forty-nine (76\%) patients had a preexisting disease, 33 $(51 \%)$ required invasive mechanical ventilation, $40(62 \%)$ were in shock and needed vasoactive drugs, and 30 (46\%) required renal replacement therapy. The ICU death rate for chikungunya was $28 \%$, slightly higher than the usual $22 \%$ ICU death rate (A. Koeltz, unpub. data). Five patients had encephalitis, 2 had myocarditis, and 4 had Guillain-Barré syndrome (GBS). Fifty-five patients had a severe form of chikungunya, and 21 had illness consistent with the case definition for severe sepsis; for 2 patients, no other cause for GBS than CHIKV was identified. Two patients had CHIKV-leptospirosis co-infection, and 1 had CHIKVdengue virus co-infection. Among the 55 patients who had the severe form of chikungunya, 17 had exacerbations of a chronic condition.

Chikungunya can be complicated by severe multiple organ failure and lead to death either from exacerbation of a preexisting disease or by severe atypical infection. Severe septic shock directly attributable to CHIKV was reported during the 2014 outbreak $(7,8)$, and these reports seem consistent with our study ( 2 cases). This finding could be explained by the fact that chikungunya induces lymphopenia.

Neurologic complications of arbovirus infections are well documented, as illustrated by the high incidence of GBS reported during French Polynesia's outbreak of Zika virus (42 cases) (9). In our study, we observed 4 severe cases of GBS, and 10 GBS cases were managed in the hospital during the outbreak; GBS incidence was 4 times higher than usually observed in this hospital.

The most severe atypical complication in our study was myocarditis ( 2 cases), which had a 100\% case-fatality rate. These deaths included an 11-year-old child and a 56-year-old woman without preexisting disease.

Our findings indicate that critical illness frequently complicates the course of CHIKV infection. Hospitals in chikungunya-endemic areas should be aware of the potential for increases in the number of ICU admissions during outbreaks.

\section{Acknowledgment}

We thank Thomas Koeltz for his review.

\section{About the Author}

Dr. Koeltz is a cardiac anesthesiologist at Bichat Claude-Bernard University Hospital in Paris. His research interests include infectious diseases in tropical areas. 
Table. Clinical and laboratory characteristics of 64 patients with chikungunya virus infection admitted into the intensive care department, French Polynesia, 2014-2015*

\begin{tabular}{|c|c|}
\hline Characteristic & Result \\
\hline \multicolumn{2}{|l|}{ Baseline } \\
\hline Median age, y (IQR) & $62(49-71)$ \\
\hline \multicolumn{2}{|l|}{ Sex, no. $(\%)$} \\
\hline $\mathrm{M}$ & $37(58)$ \\
\hline $\mathrm{F}$ & $27(42)$ \\
\hline \multicolumn{2}{|l|}{ Preexisting disease, no. (\%) } \\
\hline Hypertension & $37(58)$ \\
\hline Diabetes mellitus & $22(34)$ \\
\hline Chronic renal failure & $15(23)$ \\
\hline Chronic heart failure & $12(19)$ \\
\hline Chronic liver disease & $3(5)$ \\
\hline None & $15(23)$ \\
\hline Simplified Acute Physiology Score (IQR) & $48(28.5-68.5)$ \\
\hline \multicolumn{2}{|l|}{ Chikungunya diagnosis, no. (\%) } \\
\hline By reverse transcription PCR & $42(66)$ \\
\hline By $\lg M$ & $26(41)$ \\
\hline By reverse transcription PCR and IgM & $4(6)$ \\
\hline \multicolumn{2}{|l|}{ Finding at admission } \\
\hline \multicolumn{2}{|l|}{ Organ failure,† no. (\%) } \\
\hline Hemodynamic & $40(63)$ \\
\hline Renal & $30(47)$ \\
\hline Neurologic & $20(31)$ \\
\hline Respiratory & $33(52)$ \\
\hline Hepatic & $16(25)$ \\
\hline Hematologic & $9(14)$ \\
\hline \multicolumn{2}{|l|}{ Laboratory } \\
\hline Leukocyte count, cells $/ \mathrm{m}^{3}$, median (IQR) & $11,600(7,200-15,200)$ \\
\hline Lymphocyte count, cells $/ \mathrm{m}^{3}$, median (IQR) & $1,000(600-1,500)$ \\
\hline Lymphopenia, $<1,000$ cells $/ \mathrm{m}^{3}$ ( $\%$ of patients) & $36(56)$ \\
\hline Platelet count, cells $/ \mathrm{m}^{3}$, median (IQR) & $155,000(79-208)$ \\
\hline Platelet count, $<150,000$ cells $/ \mathrm{m}^{3}$ ( $\%$ of patients) & $34,000(53)$ \\
\hline Creatinine, $\mu \mathrm{mol} / \mathrm{L}$, median (IQR) & $132(79-184)$ \\
\hline Creatine phosphokinase, mmol/L, median (IQR) & $222(124-1,160)$ \\
\hline Alanine aminotransferase, UI/L, median (IQR) & $35(19-76)$ \\
\hline C-reactive protein, mg/L, median (IQR) & $10.6(2.8-18.3)$ \\
\hline Procalcitonin, $\mu \mathrm{g} / \mathrm{L}$, median (IQR) & $1.72(0.42-18.3)$ \\
\hline Lactate, $\mathrm{mmol} / \mathrm{L}$, median $(\mathrm{IQR})$ & $2.6(1.1-5.4)$ \\
\hline \multicolumn{2}{|l|}{ Chikungunya reverse transcription PCR $\ddagger$} \\
\hline Viral load in serum, $\log _{10}$ copies/mL (IQR) & $7.52(3.47-9.39)$ \\
\hline Viral load in cerebrospinal fluid, $\S \log _{10}$ copies/mL (IQR) & $4.18(3.86-4.26)$ \\
\hline \multicolumn{2}{|l|}{ Outcome variable } \\
\hline ICU length of stay, $d$, median (IQR) & $3(2-7)$ \\
\hline Crude intensive care unit deaths, no (\%) & $18(28)$ \\
\hline
\end{tabular}

${ }^{*} \mathrm{IQR}$, interquartile range.

†Organ failure is defined according to a Sequential Organ Failure Assessment score $\geq 3$ for each organ.

łBlood samples during the first $24 \mathrm{~h}$. Reference values are as follows: leukocytes, $4.0-10.0 \times 10^{3} \mathrm{celll} / \mathrm{mm}^{3}$; lymphocytes, $1.5-3.4 \times 10^{3}$ cells $/ \mathrm{mm}^{3}$; platelets, $150-400 \times 10^{3} / \mathrm{mm}^{3}$; creatinine, $0.56-1.0 \mathrm{mg} / \mathrm{dL}$; creatine phosphokinase, $0-130 \mathrm{U} / \mathrm{L}$; alanine aminotransferase, $0-35 \mathrm{U} / \mathrm{L}$; C-reactive protein, $<5 \mathrm{mg} / \mathrm{L}$; procalcitonin, $<0.5 \mathrm{ng} / \mathrm{mL}$; lactate, $5-15 \mathrm{mg} / \mathrm{dL}$.

$\S$ Virus load was positive for 5 of the 9 cerebrospinal fluid samples.

\section{References}

1. Nhan T-X, Claverie A, Roche C, Teissier A, Colleuil M, Baudet J-M, et al. Chikungunya virus imported into French Polynesia, 2014. Emerg Infect Dis. 2014;20:1773-4. http://dx.doi.org/10.3201/eid2010.141060

2. Nhan TX, Musso D. The burden of chikungunya in the Pacific. Clin Microbiol Infect. 2015;21:e47-8. http://dx.doi.org/10.1016/ j.cmi.2015.02.018

3. Aubry M, Teissier A, Roche C, Richard V, Yan AS, Zisou K, et al. Chikungunya outbreak, French Polynesia, 2014. Emerg Infect Dis. 2015;21:724-6. http://dx.doi.org/10.3201/ eid2104.141741

4. Dellinger RP, Carlet JM, Masur H, Gerlach H, Calandra T, Cohen J, et al.; Surviving Sepsis Campaign Management Guidelines Committee. Surviving Sepsis Campaign guidelines for management of severe sepsis and septic shock.

Crit Care Med. 2004;32:858-73. Erratum in Crit Care

Med. 2004;32:2169-70. Erratum in Crit Care Med. 2004; 32:1448. http://dx.doi.org/ 10.1097/01.CCM.0000117317. 18092.E4

5. Venkatesan A, Tunkel AR, Bloch KC, Lauring AS, Sejvar J, Bitnun A, et al.; International Encephalitis Consortium. Case definitions, diagnostic algorithms, and priorities in encephalitis: consensus statement of the International Encephalitis Consortium. Clin Infect Dis. 2013;57:1114-28. http://dx.doi.org/10.1093/cid/ cit458

6. Caforio AL, Pankuweit S, Arbustini E, Basso C, Gimeno-Blanes J, Felix SB, et al. Current state of knowledge on aetiology, diagnosis, management, and therapy of myocarditis: a position statement of the European Society of Cardiology Working Group on Myocardial 
and Pericardial Diseases. Eur Heart J. 2013;34:2636-48, 2648a-2648d.

7. Torres JR, Castro JS, Rodríguez L, Saravia V, Arvelaez J, Ríos-Fabra A, et al. Chikungunya fever: ftypical and lethal cases in the Western Hemisphere: A Venezuelan experience. IDCases. 2014;2:6-10. http://dx.doi.org/10.1016/j.idcr.2014.12.002

8. Rollé A, Schepers K, Cassadou S, Curlier E, Madeux B, Hermann-Storck C, et al. Severe sepsis and septic shock associated with chikungunya virus infection, Guadeloupe, 2014. Emerg Infect Dis. 2016;22:891-4. http://dx.doi.org/10.3201/eid2205.151449

9. Cao-Lormeau V-M, Blake A, Mons S, Lastère S, Roche C, Vanhomwegen J, et al. Guillain-Barré syndrome outbreak associated with Zika virus infection in French Polynesia: a casecontrol study. Lancet. 2016;387:1531-9. http://dx.doi.org/10.1016/ S0140-6736(16)00562-6

Address for correspondence: Adrien Koeltz, Department of Anesthesiology and Intensive Care, Centre Hospitalier Universitaire Bichat Claude-Bernard, 46 Rue Henri Huchard, 75018 Paris, France; email: adrien.koeltz@aphp.fr

\section{African Swine Fever Virus, Siberia, Russia, 2017}

\section{Denis Kolbasov, llya Titov, Sodnom Tsybanov, Andrey Gogin, Alexander Malogolovkin}

Author affiliation: Federal Research Center for Virology and Microbiology, Pokrov, Russia

DOI: https://doi.org/10.3201/eid2404.171238

African swine fever (ASF) is arguably the most dangerous and emerging swine disease worldwide. ASF is a serious problem for the swine industry. The first case of ASF in Russia was reported in 2007. We report an outbreak of ASF in Siberia, Russia, in 2017.

A frican swine fever (ASF) is arguably the most dangerous swine disease worldwide. ASF virus (ASFV) is highly virulent for domestic swine and remains a global threat because no effective vaccine is available to eradicate the disease. The emergent potential of ASF has been demonstrated by its spread into Russia. In the 10 years since ASF was first diagnosed in the Caucasian region of Russia (1), the disease has reached Palearctic regions and is spreading into western Europe $(2,3)$.

In 2017, the Federal Service for Veterinary and Phytosanitary Surveillance (Rosselkhoznadzor) reported that, during 2007-2017, >1,000 ASF outbreaks resulted in deaths of $\approx 800,000$ pigs in 46 regions across Russia (4). Production of backyard swine industry decreased by almost half, from 1,119 tons of pork in 2007 to 608 tons of pork in 2017 (5). However, highly industrialized pig farms showed increased production every year during this same period, despite the ASF epidemic.

ASF has seriously affected and is actively spread by wild boar populations in Russia, but accurate numbers of boar killed by ASF or culling attempts are difficult to estimate. In June 2017, ASF was detected in the Czech Republic in 2 wild boar ( 6 ), demonstrating disease spread toward western Europe. In 2017, ASFV cases among backyard domestic pigs were detected in July in Romania (7), and later in October 2017 in Moldova (8). We report an outbreak of ASF in Far Eastern Russia.

Early in March 2017, an ASF outbreak was reported on 1 backyard farm in the Irkutsk region near the border with Mongolia (Figure) (5). All pigs had clinical signs typical of acute ASF, and 40 pigs died within 6 days of the appearance of the first clinical signs. In a $5-\mathrm{km}$ risk zone established around the affected farm, 1,327 pigs were slaughtered within 3 days. Epidemiologic analysis showed that the farmer used table leftovers to feed pigs.

ASFV DNA was identified by real-time PCR in the frozen pork products found on the farm. The origin of contaminated pork products is still under investigation. It is likely that ASFV-contaminated pork products provided a source of infection because these products are the most common source of ASF infection on backyard farms (9). ASF outbreaks nearest to the outbreak in Irkutsk occurred $>4,000 \mathrm{~km}$ away in European Russia. Such a long geographic distance between ASF outbreaks within the country demonstrates that ASFV has a tremendous capacity for transboundary and transcontinental spread.

We identified the ASFV isolate from Irkutsk (ASFV/ Irkutsk/dom/2017) by using nucleotide sequencing and molecular analysis. This isolate has capsid protein $\mathrm{P} 72$ genotype II and central variable region I and is an intergenic region (IGR) I variant (GenBank accession nos. KY963545, KY938010, and KY982843, respectively) according to the nomenclature of Gallardo et al. (10). The intergenic region between the $I 73 R$ and $I 329 L$ genes at the right end of the ASFV/Irkutsk/dom/2017 genome contains no additional tandem-repeat sequences. The ASFV IGRI variant is identical to the ASFV/Georgia/wb/2007 index isolate of the epidemic in Georgia in 2007 but represents an ASFV variant that is rare among recent ASFV isolates in Russia. In comparison, all recent ASF outbreaks in European Russia and eastern Europe have been caused by ASFV of the IGRII variant, which has an insertion of a tandem-repeat sequence in the intergenic region between the I173R and the I329L protein genes. 